\title{
UAHSP
}

The Internet Joưnal of Allied Health Sciences and Practice

A Peer Reviewed Publication of the College of Health Care Sciences at Nova Southeastern University

Dedicated to allied health professional practice and education

http://ijahsp.nova.edu Vol. 11 No. 3 ISSN 1540-580X

\section{Is there Agreement between Self-Reported and Scale Measurements of Height and Weight?}

\author{
Christopher T. Wagner, B.S., DPT1 \\ Brittney M. Lamb, B.S., DPT 1 \\ Jared L. Graham, B.S., DPT1 \\ Paul A. Salamh, P.T., D.P.T., PhD(c)2 \\ Cheryl Hill, P.T., PhD ${ }^{3}$ \\ Morey J. Kolber, P.T., PhD, OCS 4
}

1. Doctoral Physical Therapy Student, College of Health Care Sciences, Nova Southeastern University, Ft. Lauderdale, Florida

2. Director of Physical Therapy, Southeastern Orthopedics Physical Therapy, Raleigh, North Carolina

3. Professor, Physical Therapy Department, College of Health Care Sciences, Nova Southeastern University, Ft. Lauderdale, Florida

4. Associate Professor, Physical Therapy Department, College of Health Care Sciences, Nova Southeastern University, Ft. Lauderdale, Florida

United States

CITATION: Wagner C, Lamb B, Graham J, Salamh P, Hill C, Kolber M. Is there Agreement Between Self-Reported and Scale Measurements of Height and Weight? The Internet Journal of Allied Health Sciences and Practice. July 2013. Volume 11 Number 3.

\section{ABSTRACT}

Background: The assessment of height and weight (body mass) are common components of the medical examination, often requisite subject characteristics collected for research, and frequently used in the writing of life and medical insurance policies. These measurements may be gathered by actual measurement procedures or through patient self-report. Purpose: The purposes of this study were to 1) determine if significant differences exist between self-reported and measured height and body mass, 2) determine the influence of gender on differences between self-reported and actual measurements, and 3) determine the range of differences (levels of agreement) that may exist between self-reported and actual measurements. Subjects: A convenience sample of one hundred and two participants, 48 men and 54 women, were recruited from a local university setting. Methods: Participants documented their perceived height and mass on a standardized questionnaire. This was then compared to formal measurements taken using the Cardinal/DETECTO 439 3P series scale. Results: Statistically significant differences were present between self-report and measured height and body mass when total participants were analyzed independent of gender $(p \leq .009)$. When gender was analyzed independently, significant differences were identified for men $(p \leq .004)$ for both height and body mass, whereas significant differences for women existed only when comparing body mass $(p=.038)$. A trend existed for over-reporting height and under-reporting body mass. Conclusion: Although self-reported measurements are an efficient means to capture anthropometric data, evidence suggests that there may be substantial differences in self-reports versus actual measurements.

\section{INTRODUCTION}

The assessment of height and weight (body mass) are integral anthropometric components of the medical examination. Moreover, height and body mass are often requisite subject characteristics collected for research studies and with the acquisition of services and products such as life and medical insurance. These measurements may be gathered by actual measurement 
procedures or through self-report that is based on one's recall and self-measurement. Self-report of height and body mass may be used in clinical practice as well as in research and epidemiological studies because they provide a quick, inexpensive, and simple method of collecting data. ${ }^{1-3}$

Although self-reported documentation of height and body mass offer a quick and easy method to collect anthropometric data, inherent limitations may exist in one's self-report versus actual values. Previous studies suggest that the accuracy of self-report may vary according to age, gender, ethnic background, education level, morphology, disease history/health, and socioeconomic status. ${ }^{3-18}$ Although there are potential covariates that may affect the accuracy of self-reported measures, a majority of studies have reported good reliability between self-reported and measured body mass and height with values ranging from 0.83 to $0.98 .7,9,10,13,15,19$ Despite finding good reliability between self -reports and direct measurements, a trend for individuals to underestimate their body mass and overestimate their height has been identified. $5,8,9,14,18,19$

Underestimating body mass values is generally more prevalent among women, whereas overestimating height and body mass is more prevalent among men. 3,5,8,9,9,20 When gender was analyzed individually, prior research has suggested body mass discrepancies ranging from $-1.50 \mathrm{~kg}$ to $0.93 \mathrm{~kg}$ for men and from $-1.80 \mathrm{~kg}$ to $0.81 \mathrm{~kg}$ for women.4,6,8,9,13,15,19 Differences in height values ranged from $0.90 \mathrm{~cm}$ to $1.54 \mathrm{~cm}$ for men and from $0.37 \mathrm{~cm}$ to $4.0 \mathrm{~cm}$ for women. $4,6,8,9,13,15,19$ Potential limitations of previous investigations include failure to document or ensure calibration of the scale, the time span between the assessed and self-reported measurements, failure to use a bubble level on the stadiometer to ensure a consistent angle for measuring height, and the use of multiple researchers with non-specific procedures to record the anthropometric measurements (which can affect the reliability of the measured values).6,8-10, 13,15-17,19-20 Additional limitations include the omission of blinded investigators and/or participants and a sample size that is restricted only to women. $6,8,10,15$ Numerous studies have investigated the reliability of selfreported anthropometric measures using the most common statistical approaches including, but not limited to, correlation coefficients, regression lines, and the comparison of means. ${ }^{6-8,10-12,15,17,19,20}$ However, the aforementioned studies have failed to calculate limits of agreement. Conversely, there are very few studies that have used the Bland-Altman approach to graphically represent the limits of agreement. 4,9,13,18,21 According to Bland and Altman, widely used statistical approaches can be misleading and can guide researchers to erroneous conclusions; therefore, it is suggested that data be analyzed using an alternative approach that includes calculating limits of agreement to ensure accuracy of the overall level of agreement. ${ }^{21,22}$

Previous researchers have assessed the relationship between self-reported and measured height and body mass values using a variety of data collection methods; however, the accuracy of self-reported body mass and height has been questioned when used for individual purposes such as clinical decision making. ${ }^{5-10,12-15,18-23}$ Therefore, the purpose of this study was to determine if significant differences exists between self-reported height and mass and actual measured height and body mass. In addition, it was sought to determine the level of agreement (range of differences) that may exist between self-reported and actual measurements. Lastly, it was sought to determine if gender influences the accurate perception of height and mass.

\section{DESIGN}

\section{Participants}

A convenience sample of one hundred and two college age students, 48 men and 54 women, were recruited from a local university setting. Participants who met study requirements were provided with an informed consent document approved by the Institutional Review Board at Nova Southeastern University, and all questions were answered to their satisfaction prior to commencing data collection. An a priori power analysis indicated that a sample of 99 would be required to achieve $80 \%$ power if it was posit for an effect size of .40 at the $\alpha_{2}-.05$ level of significance.

Participants completed a questionnaire to report age, height, body mass, as well as their most recent use of a scale to measure their height or body mass. Inclusion criteria consisted of individuals who were at least 18 years of age, had sufficient comprehension of the English language as necessary to read forms and communicate with investigators, ability to step on to a scale independently, and individuals who did not exceed the operating parameters of the scale (height and body mass).

\section{Instruments}

The Cardinal / DETECTO 439 3P series scale (Cardinal Scale Manufacturing, Webb City, MO, Figure 1) was used to measure height and body mass. The manufacturer's margin of error (tolerance) for mass varies depending on the amount of mass present on the scale. For example, with no mass on the scale, the margin of error is $+/-50$ grams $(\mathrm{g})$. This margin remains until a mass load of 60 kilograms $(\mathrm{kg})$ which increases the margin of error to $+/-75 \mathrm{~g}$. The margin increases slightly until the scale reaches capacity of $181 \mathrm{~kg}$ with a margin of error of $+/-350 \mathrm{~g}$. These margins are acceptable because of the very low amount of mass present in each margin and because of our patient population being in the lower, more accurate mass loads. The scales maximum tolerance for mass is $181.6 \mathrm{~kg}$ (400lbs $4 \mathrm{oz}$ ). The manufacturer's margin of error (measuring tolerance) for the height

(c) The Internet Journal of Allied Health Sciences and Practice, 2013 
rod is $+/-1 / 8$ inches. The scale has both a minimum and maximum height tolerance. The minimum height that could be recorded was 76.2 centimeters $(\mathrm{cm})(2 \mathrm{ft} 6 \mathrm{in}$.). The maximum height that could be recorded was $198.12 \mathrm{~cm}(6 \mathrm{ft} 6 \mathrm{in}$.)

\section{Procedures}

All participants were provided with a Nova Southeastern University Institutional Review board approved consent form prior to their participation. Three investigators were utilized in this investigation to ensure blinding of data. Investigator 1 provided the agreeing participants appropriate paperwork and gained consent from each participant. The participant was then brought to a private area where Investigator 1 collected demographic data which included the participants' self-report of height and mass. Participants were then instructed to remove shoes, socks, and shirt. Women participating were asked to wear a sports bra or bathing suit top. Shorts were required for all measurements taken. The scale (See Figure $1 \& 2$ ) was used for all measurements and was calibrated immediately prior to the initiation of data collection.

Figure 1. Cardinal / DETECTO 439 3P Series Scale

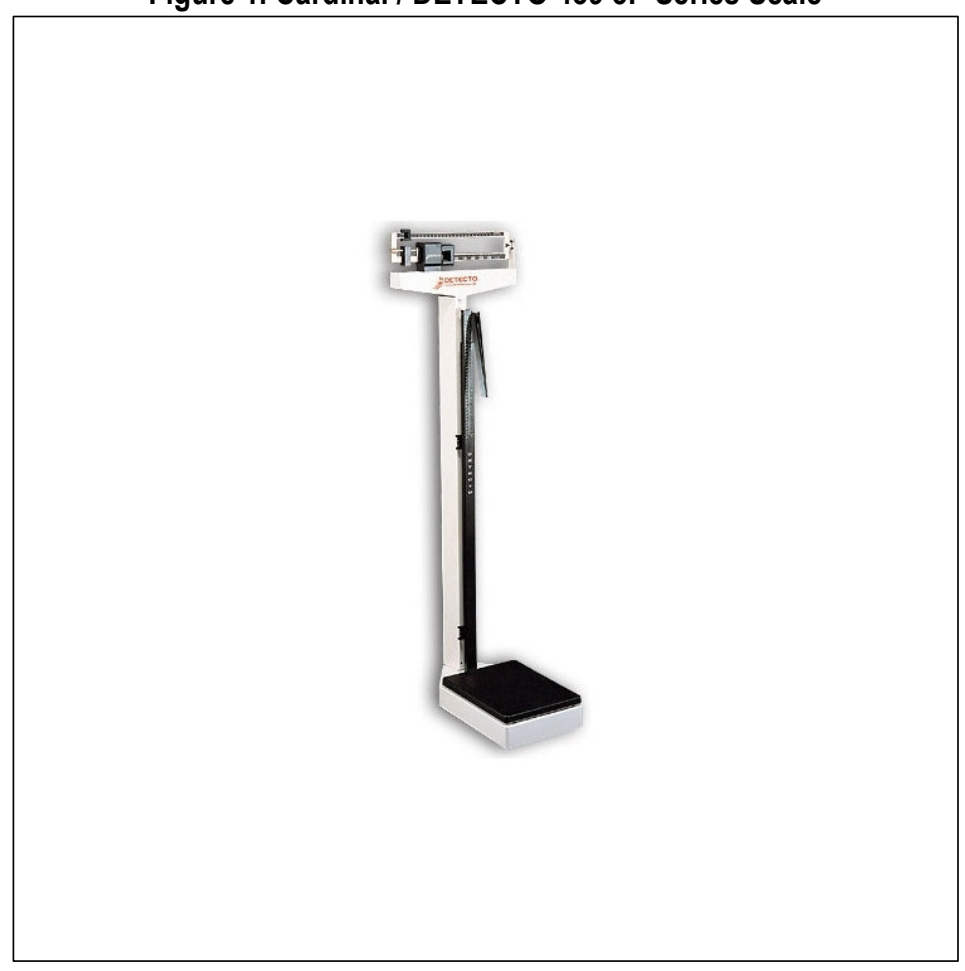

Figure 2. Cardinal / DETECTO 439 3P Series Scale Illustrating Measurement Regions

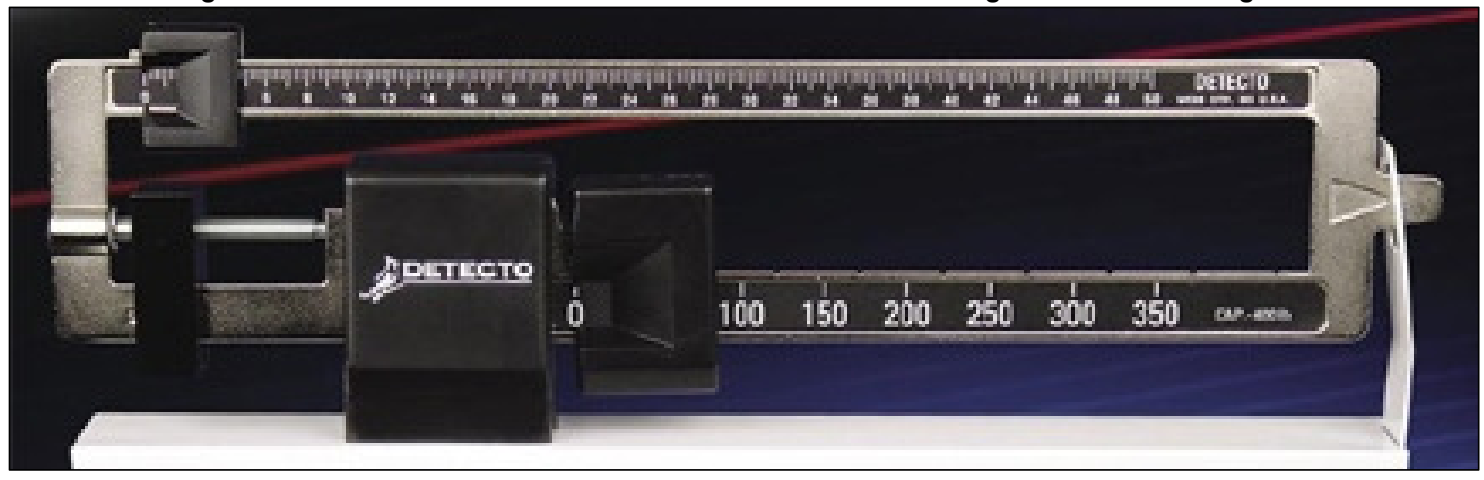

Investigators 2 and 3 measured height and weight according to the scale manufacturer's instructions in the operating manual \# 8525-M063-01 Rev H 11/10. Both investigators 2 and 3 were blinded to the self-report data. A bubble level (See Figure 3) was installed on the stadiometer to ensure a true horizontal measurement. 
Figure 3. Stadiometer with Bubble Level

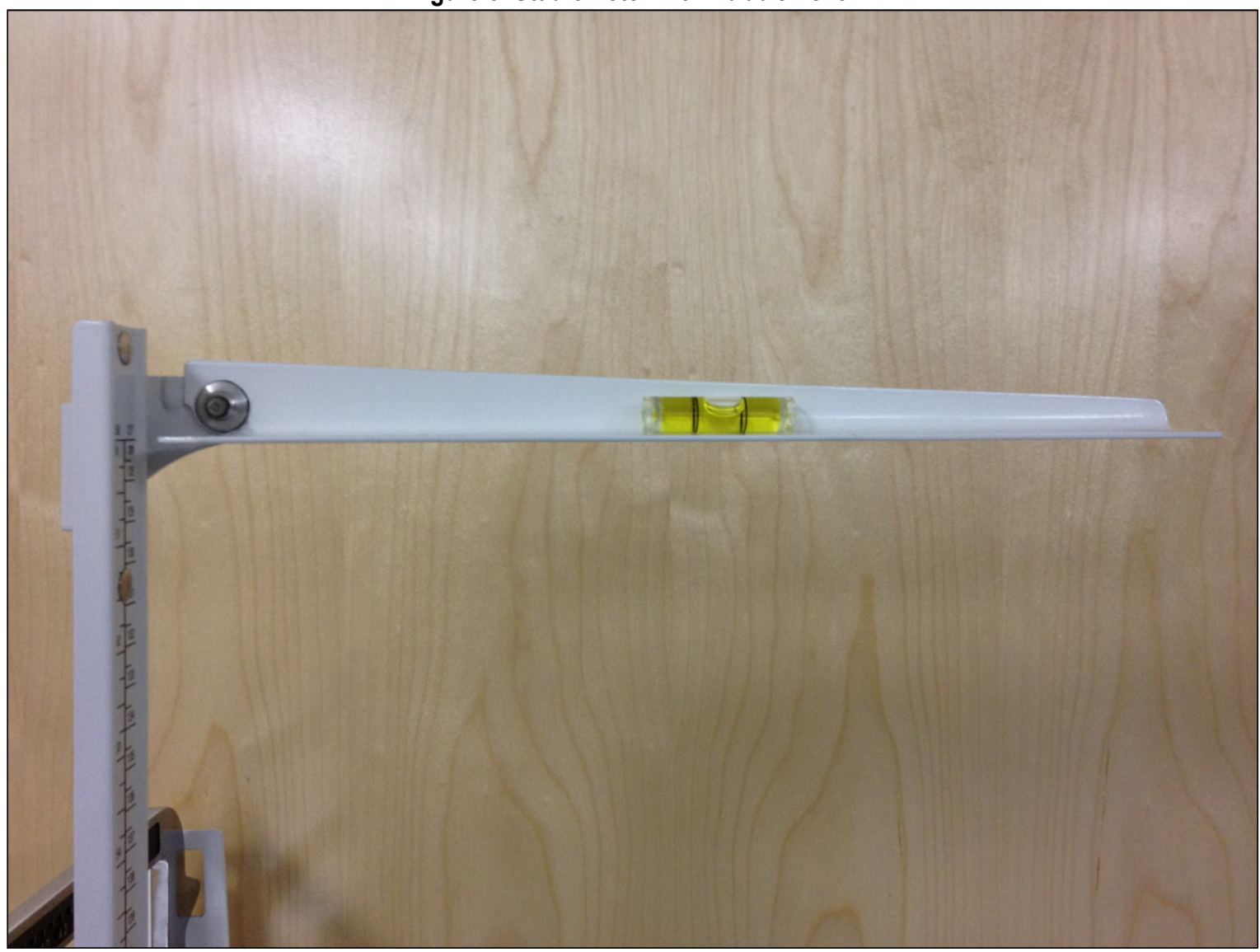

After completion of height and body mass measurements Investigator 1, who obtained the consent, transferred measurements to a data collection form. The total time including consent was 15 minutes. The first thirty participants were requested to participate in an intrasession intrarater reliability trial of the height and weight measurements. The repeat measurement took place 30 minutes later.

\section{DATA ANALYSIS}

Data analysis was performed with SPSS version 20.0 for Windows statistical program. The mean and standard deviation (SD) were calculated for descriptive data which included age, measured-height, measured-body mass, and calculated body mass index (BMI). The intrasession test-retest reliability of measurements for both height and body mass using the scale were performed on the first 30-participants and determined by the ICC Model 3, k. Interpretation of ICC values was based on guidelines offered by Portney and Watkins, where a value above 0.75 was classified as good reliability. ICC values may be influenced by intersubject variability of scores because a large ICC may be reported despite poor trial-to-trial consistency if the intersubject variability is too high. ${ }^{24}$ The standard error of measurement (SEM) is not affected by intersubject variability. ${ }^{24}$ Therefore, SEM was reported in conjunction with the ICC's.

Differences between self-reported and scale measured height and body mass were analyzed using a paired t-test with significance set at $\alpha_{2}=.05$. Comparison was made for total participants and based on gender grouping (men and women). Additionally, $95 \%$ limits of agreement (LOA) were calculated to determine where $95 \%$ of differences between self-reported and scale measurements would fall for total participants as well as gender. The $95 \%$ LOA were calculated using the formula: $95 \%$ limits of agreement $=$ mean difference $+/-2 S D .24$

\section{RESULTS}

A total of 102 participants (48 men and 54 women) completed the study. The calculated mean and standard deviation (SD) for the participants' age, height, body mass, and body mass index were $26( \pm 3.9)$ years, $171( \pm 9.7) \mathrm{cm}, 71( \pm 14.4) \mathrm{kg}$, and $24( \pm 3.4)$ $\mathrm{kg} / \mathrm{m} 2$ respectively, which is considered to be a reasonably healthy category. All of the participants were either enrolled in a 
graduate college curriculum or served a faculty role at the site of data collection. Ninety-five percent of participants were Caucasian. Forty-seven percent of participants reported weighing themselves in the past week compared to only one percent of participants who reported measuring their height in the past week.

Descriptive data including the mean (SD) of height and body mass is presented in Table 1. A trend existed for participants overreporting height and under-reporting body mass. When comparing self-report to scale measurements of height and body mass (Table 2), statistically significant differences were present when total participants were analyzed independent of gender $(p \leq$ .009). When gender was analyzed individually, significant differences in height and body mass were identified for men ( $p \leq .004)$; however, significant differences for women were only found when comparing body mass $(p=.038)$.

\begin{tabular}{|l|c|c|c|c|}
\hline \multicolumn{5}{|c|}{ Table 1: Descriptive Height and Body Mass Data } \\
\hline & $\begin{array}{c}\text { Self-Report } \\
\text { Height (cm) } \\
\text { Mean(SD) }\end{array}$ & $\begin{array}{c}\text { Scale measured } \\
\text { Height (cm) } \\
\text { Mean(SD) }\end{array}$ & $\begin{array}{c}\text { Self-Report } \\
\text { Body mass(kg) } \\
\text { Mean(SD) }\end{array}$ & $\begin{array}{c}\text { Scale measured } \\
\text { Body mass(kg) } \\
\text { Mean(SD) }\end{array}$ \\
\hline Total (102) & $171.5( \pm 9.7)$ & $170.6( \pm 9.6)$ & $71.4( \pm 14.4)$ & $72.0( \pm 14.7)$ \\
\hline Men (48) & $178.2( \pm 6.9)$ & $177.4( \pm 7.0)$ & $81.8( \pm 11.1)$ & $82.5( \pm 10.8)$ \\
\hline Women (54) & $165.4( \pm 7.6)$ & $164.6( \pm 7.3)$ & $62.1( \pm 10.1)$ & $62.7( \pm 10.8)$ \\
\hline
\end{tabular}

$\mathrm{SD}=$ standard deviation; $\mathrm{cm}=$ centimeter $; \mathrm{kg}=$ kilogram

An intraclass correlation coefficient (ICC-3,k) of 0.99 for both height and body mass was present. The standard error of measurement for scale measurements of height and body mass were $1.1 \mathrm{~cm}$ and $1.4 \mathrm{~kg}$ respectively.

In regard to agreement (Table 2), 95\% LOA indicate that differences in self-reported height can vary between $7.39 \mathrm{~cm}$ less than and $9.09 \mathrm{~cm}$ greater than scale measurements, whereas body mass self reports may be $4.46 \mathrm{~kg}$ less than and $2.93 \mathrm{~kg}$ greater than scale measurements. Generally speaking, participants had a greater trend toward under-reporting weight and over-reporting height.

Table 2: Comparison Data Analysis Self-Report and Scale Measurements

\begin{tabular}{|l|c|c|c|}
\hline \multicolumn{2}{|l|}{} & Height (cm) & Body mass(kg) \\
\hline Total (102) & $p$-value* & $.009 \dagger$ & $\leq .001 \dagger$ \\
& $95 \%$ LOA & -5.47 to 7.14 & -4.16 to 2.93 \\
\hline Men (48) & $p$-value* & $\leq .001 \dagger$ & $.004 \dagger$ \\
& $95 \%$ LOA & -2.11 to 3.77 & -3.82 to 2.46 \\
\hline Women (54) & p-value* & .139 & $.038 \dagger$ \\
& $95 \%$ LOA & -7.39 to 9.09 & -4.46 to 3.34 \\
\hline
\end{tabular}

${ }^{*}$ Paired t-test; LOA = Levels of agreement at $95 \%$ confidence interval; $\uparrow=$ statistically significant; $\mathrm{cm}=$ centimeter; $\mathrm{kg}=$ kilogram

\section{DISCUSSION}

The overall results of this study were similar to previous investigations comparing self-reported height and weight to actual measurements. $1,2,5$ The results of our study show that self-reported height and body mass when given by college students are significantly different than actual measurements, and the trend to over/under-report height and body mass exceeds the scale's threshold of error. In regards to range differences, this study showed that individual differences in height could range from 7.39 $\mathrm{cm}$ shorter to $9.09 \mathrm{~cm}$ taller than self- reported and body mass could range from $4.46 \mathrm{~kg}$ lighter to $3.34 \mathrm{~kg}$ heavier than selfreported values. This is important when considering clinical risk factors for conditions such as cardiovascular disease and diabetes as well as body mass decisions that may be made based on self-report. Unfortunately, previous studies appear to have only calculated comparisons of overall means and not $95 \%$ LOA, thus no data were identified to compare the findings of this study. Statistics that were used in those previous studies had the objective to differentiate gender, ethnic background, age, BMI, educational level, etc. from other categories, all comparing data to the overall mean and not on the actual range of agreement 
(differences) between self-reported height and body mass. It should be recognized that while a body of evidence exists to substantiate a correlation between self-reported and actual measurements, differences in height and body mass may exist and should be considered when making evaluating one's individual anthropometrics.

When considering the influence of gender on differences in self-reported and actual measurements of body mass it was found that both men and women tend to underreport body mass. On average, self-reported body mass was less than the scalemeasured weight: $0.7 \mathrm{~kg}$ for men and $0.6 \mathrm{~kg}$ for women. Our results are in agreement with previous studies that identified a greater trend for participants of both genders to under-report body mass. $5,8,9,14,18,19$ Conversely, it was found that while selfreported height for both men and women were $0.8 \mathrm{~cm}$ higher than scale measured height, significant differences were only found for men who tended to over report their height. In contrast, Bolton-Smith et al described an under-reporting of both height and body mass; however, their study focused on an older population in Scotland. ${ }^{3}$

When analyzing men and women as one group, the differences in self-reported and actual measurements of height and body mass appear to be lower $(0.9 \mathrm{~cm}$ and $0.6 \mathrm{~kg}$ respectively) than similar studies which have reported height being overestimated as much as $5.0 \mathrm{~cm}$ and weight being underestimated as much as $2.7 \mathrm{~kg} .7,7,10,13,15,19$ However, previous studies did not specifically recruit college age individuals as in this study. These results may be a result of societal tendencies and influenced by culture, media, perception of body image, and other factors. With younger subject studies, the agreement between self- reported height and mass are thought to be more accurate when compared to studies performed on specific groups such as the elderly, those with abnormal BMI (over abundant and under abundant), individuals with lower education, and restrained eaters (dieting).3-13, 15$16,18,20$

The use of self-reported measures is often used in order to keep research and or census costs low. The use of self-reported values would be appropriate to implement when research or census cost must remain low because of the low cost of questionnaire-based self-report studies. A difference in methodology between the current study and previous investigations may lie in the procedures used for the scale measurement. The reliability of scale measurements of height and body mass in our study was ICC $=0.99$ for both height and weight, which is considered excellent. Specifically, a bubble attachment to the stadiometer arm was utilized to insure uniform measurement angle of height, calibrated the scale to manufacturer's standards, and used specific measurement procedures throughout all measurements of this study.

\section{LIMITATION AND FUTURE INVESTIGATIONS}

The subject sample that was used in this study was limited in culture/ethnicity, age, educational background as all were enrolled in college, and BMl; thus, it may not represent the heterogeneous general population. The subject sample was made up of mainly Caucasian participants with an average age of 26 years ( $3.9 \mathrm{SD}$ ) and an average BMl of $24 \mathrm{~kg} / \mathrm{m}^{2}$, which is considered to be a reasonably healthy category. As seen in other studies, most subject samples were of older subjects with BMI ranging in the obese category. Another limitation to our study is the use of an analog scale. In previous studies, when digital scales have been used, a lower margin of error is achieved because of the lower tolerance present in digital scales when compared to analog scales.10,16 The Cardinal/DETECTO $4393 \mathrm{P}$ series scales were chosen as these scales are readily accessible in most clinics and research laboratories.

\section{CONCLUSION}

In this study, self-reported height and body mass were significantly different than measured height and body mass. Individual differences in height ranged from $7.39 \mathrm{~cm}$ shorter to $9.09 \mathrm{~cm}$ taller than self- reported and body mass ranged from $4.46 \mathrm{~kg}$ lighter to $3.34 \mathrm{~kg}$ heavier than self-reported values. This current investigation also revealed that gender influenced height and body mass when comparing self-reported measurements to actual measurements. As noted in previous studies, self-reported values would be appropriate to implement when research or census cost must remain low, because of the low cost of questionnairebased self-report studies or when individual differences are not relevant. However, as seen in this study as well as suggested in several studies, individual differences may vary and have an increased range both over- and under-estimating. Other studies have shown that culture, age, economic status, body image perception, education, and activity level may skew self-reports of height and body mass. Although self-reported measurements are efficient means to capture such data, evidence from this study suggests that there may be substantial differences in self-report versus actual measurements.

\section{REFERENCES}

1. Black DR, Taylor AM, Coster DC. Accuracy of self-reported body weight: Stepped Approach Model component assessment. Health Educ Res. Jun 1998;13(2):301-7. [PMID 10181028]

2. Fonseca Mde J, Faerstein E, Chor D, Lopes CS. [Validity of self-reported weight and height and the body mass index within the "Pro-saude" study]. Rev Saude Publica. Jun 2004;38(3):392-8. [PMID 15243669] 
3. Bolton-Smith C, Woodward M, Tunstall-Pedoe H, Morrison C. Accuracy of the estimated prevalence of obesity from self reported height and weight in an adult Scottish population. J Epidemiol Community Health. Feb 2000;54(2):143-8. [PMID 10715748]

4. Taylor AW, Dal Grande E, Gill TK, et al. How valid are self-reported height and weight? A comparison between CATI selfreport and clinic measurements using a large cohort study. Aust N Z J Public Health. Jun 2006;30(3):238-46. [PMID 16800200]

5. Niedhammer I, Bugel I, Bonenfant S, Goldberg M, Leclerc A. Validity of self-reported weight and height in the French GAZEL cohort. Int J Obes Relat Metab Disord. Sep 2000;24(9):1111-8. [PMID 11033979]

6. Craig BM, Adams AK. Accuracy of body mass index categories based on self-reported height and weight among women in the United States. Matern Child Health J. Jul 2009;13(4):489-96. [PMID 18607705]

7. Davis H, Gergen PJ. The weights and heights of Mexican-American adolescents: the accuracy of self-reports. Am J Public Health. Mar 1994;84(3):459-62. [PMID 8129066]

8. Brunner Huber LR. Validity of self-reported height and weight in women of reproductive age. Matern Child Health J. Mar 2007;11(2):137-44. [PMID 17066316]

9. Lucca A, Moura EC. Validity and reliability of self-reported weight, height and body mass index from telephone interviews. Cad Saude Publica. Jan 2010;26(1):110-22. [PMID 20209215]

10. Lee DH, Shin A, Kim J, Yoo KY, Sung J. Validity of self-reported height and weight in a Korean population. J Epidemiol. 2011;21(1):30-6.[PMID 20953091]

11. Stommel M, Schoenborn CA. Accuracy and usefulness of BMl measures based on self-reported weight and height: findings from the NHANES \& NHIS 2001-2006. BMC Public Health. 2009;9:421. [PMID 19922675]

12. Stunkard AJ, Albaum JM. The accuracy of self-reported weights. Am J Clin Nutr. Aug 1981;34(8):1593-9. [PMID 7270483]

13. McAdams MA, Van Dam RM, Hu FB. Comparison of self-reported and measured BMl as correlates of disease markers in US adults. Obesity (Silver Spring). Jan 2007;15(1):188-96. [PMID 17228047]

14. Schmidt MI, Duncan BB, Tavares M, Polanczyk CA, Pellanda L, Zimmer PM. Validity of self-reported weight--a study of urban Brazilian adults. Rev Saude Publica. Aug 1993;27(4):271-6. [PMID 8209159]

15. Peixoto Mdo R, Benicio MH, Jardim PC. [Validity of self-reported weight and height: the Goiania study, Brazil]. Rev Saude Publica. Dec 2006;40(6):1065-72. [PMID 17173164]

16. Shapiro JR, Anderson DA. The effects of restraint, gender, and body mass index on the accuracy of self-reported weight. Int J Eat Disord. Jul 2003;34(1):177-80. [PMID 12772184]

17. Gillum RF, Sempos CT. Ethnic variation in validity of classification of overweight and obesity using self-reported weight and height in American women and men: the Third National Health and Nutrition Examination Survey. Nutr J. 2005;4:27. [PMID 16209706]

18. Gunnell $D$, Berney L, Holland $P$, et al. How accurately are height, weight and leg length reported by the elderly, and how closely are they related to measurements recorded in childhood? Int J Epidemiol. Jun 2000;29(3):456-64. [PMID 10869317]

19. Lim LL, Seubsman SA, Sleigh A. Validity of self-reported weight, height, and body mass index among university students in Thailand: Implications for population studies of obesity in developing countries. Popul Health Metr. 2009;7:15. [PMID 19778455]

20. Dahl AK, Hassing LB, Fransson El, Pedersen NL. Agreement between self-reported and measured height, weight and body mass index in old age--a longitudinal study with 20 years of follow-up. Age Ageing. Jul 2010;39(4):445-51. [PMID 20453247]

21. Bland JM, Altman DG. Statistical methods for assessing agreement between two methods of clinical measurement. Lancet. Feb 8 1986;1(8476):307-10. [PMID 2868172]

22. Bland JM, Altman DG. Agreed statistics: measurement method comparison. Anesthesiology. Jan 2012;116(1):182-5. [PMID 22129533]

23. Kuczmarski MF, Kuczmarski RJ, Najjar M. Effects of age on validity of self-reported height, weight, and body mass index: findings from the Third National Health and Nutrition Examination Survey, 1988-1994. J Am Diet Assoc. Jan 2001;101(1):28-34; quiz 35-26. [PMID 11209581]

24. Portney LG, Watkins MP. Foundations of Clinical Research: Applications to Practice. 3rd ed. Upper Saddle River, N.J.: Pearson/Prentice Hall, 2009.

\section{KEY TERMS}

Anthropometrics, Bodyweight, Height, Scale, Weight, Mass 